11 Ministry of Health of the People's Republic of China. National Tuberculosis Control Programme (2006-2010). Beijing, Ministry of Health, 2006.

12 Chen S, Liu B, Wang X, et al. Analysis of human resource situation about TB control in Zhejiang. Chin J Antituberc 2006; 28: 345-347.

13 Zhao Y, et al. National survey of drug-resistant tuberculosis in China. N Engl J Med 2012; 366: 2161-2170.

$14 \mathrm{Li} \mathrm{W}$, Sun Q, Yan Y, et al. Investigation of the second line anti TB drugs usage in specialist hospitals and tuberculosis control institutions. Chin J Antituberc 2010; 32: 706-709.

15 Liu X, Wang L, Du Y, et al. Current status of case referring and treatment of tuberculosis inpatients in four tuberculosis specialist hospitals in China. Chin J Antituber 2010; 32: 775-778.

\title{
First independent evaluation of QuantiFERON-TB Plus performance
}

\author{
To the Editor:
}

Tuberculosis elimination requires an effective strategy to diagnose and treat people infected with Mycobacterium tuberculosis who would otherwise be at high risk of developing and transmitting active disease $[1,2]$. The diagnostic tools for latent tuberculosis infection (LTBI) are the tuberculin skin test (TST) and the T-cell interferon- $\gamma$ release assays (IGRAs). Two IGRAs are commercially available, QuantiFERON-TB Gold In-Tube (QFT-GIT) (Qiagen, Hilden, Germany) and T-SPOT.TB (Oxford Immunotec, Abingdon, UK). Compared to the TST, IGRAs offer operational advantages and higher specificity in the bacille Calmette-Guérin (BCG)-vaccinated population [3], and they are at least as sensitive for LTBI [4]. However, IGRAs have limitations: reduced sensitivity in children and immunocompromised subjects, including HIV-infected individuals [3, 4]; failure to discriminate between active tuberculosis and LTBI; and poor correlation with the risk of progression to active disease [3].

QuantiFERON-TB Plus (QFT-Plus) is a new generation of QTF-GIT [5] that includes an additional antigen tube (TB2). The TB1 tube contains ESAT-6- and CFP-10-derived peptides (TB7.7, present in QFT-GIT, has been removed), designed to elicit cell-mediated immune responses from $\mathrm{CD} 4{ }^{+} \mathrm{T}$-helper lymphocytes. TB2 contains newly designed, shorter peptides able to stimulate interferon- $\gamma$ production by both $\mathrm{CD}^{+}$and $\mathrm{CD}^{+}$T-cells. Previous studies have reported higher $M$. tuberculosis-specific CD8 ${ }^{+}$T-cell responses in those with active tuberculosis disease compared to LTBI [6] and in those with recent $M$. tuberculosis exposure [7]. In addition, $M$. tuberculosis-specific $\mathrm{CD}^{+}$T-cells producing interferon- $\gamma$ have been detected in active tuberculosis subjects with HIV co-infection [8] and in young children with TB disease [9].

IGRAs primarily serve as tests for LTBI in clinical practice and, to date, they have no place in active tuberculosis diagnosis [10]. In the absence of a gold-standard test for LTBI against which to compare test accuracy, sensitivity and specificity are estimated using surrogate reference standards. Sensitivity is estimated in confirmed tuberculosis cases, while specificity is estimated in low-risk individuals with no known tuberculosis exposure in low-incidence settings. From November 2014 to September 2015, 119 consecutive individuals with active tuberculosis and 106 low-risk controls were enrolled and tested with QFT-Plus at different sites in Italy (Villa Marelli-Niguarda Hospital and San Raffaele Hospital, Milan; INMI, Rome; Sant'Orsola-Malpighi University Hospital, Bologna; and San Matteo University Hospital, Pavia) and one in the UK (Royal Free Hospital, London). The active tuberculosis group included adult patients (aged $\geqslant 18$ years) with microbiologically confirmed tuberculosis (either by nucleic acid amplification or culture), who had received $<15$ days of anti-tuberculosis drugs. HIV co-infected individuals and immunocompromised patients were not excluded. Additional information was collected regarding: results of QFT-GIT, BCG vaccination status, and demographic, clinical and microbiological data. Healthy controls were recruited among students at the time of enrolment at the University Vita-Salute in Milan (low-risk population, non-BCG vaccinated); all subjects were interviewed and screened for the absence of any risk factors for M. tuberculosis exposure. The study was approved by the ethical committees of all the participating centres and informed consent was obtained from study subjects before blood sample collection.

QFT-Plus kits were donated by Qiagen and used according to the manufacturer's instructions [5]. Experiments were performed in three different laboratories (San Raffaele Hospital, Sant'Orsola-Malpighi 
University Hospital and the Royal Free Hospital) by trained staff. Levels of interferon- $\gamma$ quantified by ELISA were converted to international units per millilitre using a standard curve constructed from the QFT-Plus Analysis Software provided with the kit. Test results were interpreted according to manufacturer's criteria [5]. Positivity of a single antigen tube (TB1 or TB2) was sufficient to record the QFT-Plus as positive.

Results are reported in table 1 . In the active tuberculosis group, three (2.52\%) out of 119 patients had indeterminate results. Among the $116 \mathrm{~TB}$ patients who had valid test results, 102 were positive, leading to a sensitivity in patients with active tuberculosis disease of $87.93 \%$ (95\% CI 80.76-92.67\%). 95 out of 116 were positive for TB1 and TB2, one patient was positive for TB1 only, and six patients were positive for TB2 only.

Of the 106 low-risk control subjects, three had a positive QFT-Plus test, giving a specificity of 97.17\% (95\% CI 92.01-99.03\%) in this non-BCG-vaccinated population. Two of these three positive results were positive in one antigen tube only (one in the TB1 and one in the TB2), with interferon- $\gamma$ values close to the cut- off.

Considering the quantitative data, the overall TB2 antigen interferon- $\gamma$ response was higher than in TB1 (median (interquartile range): TB1 2.09 (0.83-6.52), TB2 $2.88(1-7.89) \mathrm{IU} \cdot \mathrm{mL}^{-1}$; Wilcoxon test p=0.0002). TB2 interferon- $\gamma$ values were greater than TB1 for $63.16 \%$ (72 out of 114 ; for five patients, the exact value was not available) of the tuberculosis patients and $44.34 \%$ (47 out of 106) of the low-risk control group.

As the TB1 tube elicits a $\mathrm{CD} 4^{+} \mathrm{T}$-cell response while the $\mathrm{TB} 2$ tube elicits both a $\mathrm{CD} 4^{+}$and a $\mathrm{CD} 8^{+} \mathrm{T}$-cell response, the difference between these tubes might provide a surrogate marker of the magnitude of $\mathrm{CD} 8^{+}$ $\mathrm{T}$-cell responses. In active tuberculosis patients, we found that this difference was higher in smear-positive than smear-negative patients (Mann-Whitney test $\mathrm{p}=0.0135$ ).

Results of the commercially available QFT-GIT were available for 73 of the active tuberculosis group subjects. The head-to-head comparison of the QFT-GIT and the QFT-Plus in this group demonstrated agreement in 68 results. Four patients scored positive only with QFT-Plus (three of them scored positive for the TB2 antigens only) and one patient was QFT-GIT positive but QFT-Plus indeterminate.

This cross-sectional, multicentre study is the first independent assessment of the performance characteristics of QFT-Plus. Estimated diagnostic sensitivity (88\% including all patients) was higher than the upper confidence limit reported among the culture-confirmed, active tuberculosis patients in the most recent meta-analysis for QFT-GIT [3], suggesting that the QFT-Plus does indeed offer improved sensitivity. However, the performance characteristics of this test remain insufficient for use as a rule-out test for active tuberculosis.

Immunocompromised patients were not excluded from the study population. Four HIV/tuberculosis co-infected subjects all had a positive QFT-Plus test. Among the five patients with cancer undergoing chemotherapy, we found one negative result and one indeterminate. A negative result was also observed in

TABLE 1 QuantiFERON-TB Plus (QFT-Plus) performance characteristics in different study groups

\begin{tabular}{|c|c|c|c|c|c|c|c|c|c|}
\hline & \multirow[t]{2}{*}{ Frequency } & \multicolumn{3}{|c|}{ QFT-Plus result } & \multicolumn{2}{|c|}{$\begin{array}{l}\text { Positive results } \\
\text { in each tube }\end{array}$} & \multicolumn{2}{|c|}{ IFN- $\gamma$ concentration ${ }^{\rrbracket} \mathrm{IU} \cdot \mathrm{mL}^{-1}$} & \multirow[t]{2}{*}{$\begin{array}{l}\text { TB2-TB1 } \\
\text { IU.mL } \mathrm{mL}^{-1}\end{array}$} \\
\hline & & Indeterminate & Negative ${ }^{\#}$ & Positive ${ }^{\#}$ & TB1 & TB2 & TB1 & TB2 & \\
\hline Active TB & 119 & 3 & 14 (12.07) & 102 (87.93) & 96 & 101 & $2.09(0.83-6.52)$ & $2.88(1-7.89)$ & $0.14(-0.13-0.79)$ \\
\hline \multicolumn{10}{|l|}{ Sex } \\
\hline Male & 72 & 1 & $7(9.86)$ & $64(90.14)$ & 59 & 64 & $2.08(0.86-6.38)$ & $2.91(1.17-7.62)$ & $0.23(-0.14-0.88)$ \\
\hline Negative & 65 & 1 & 12 (18.75) & 52 (81.25) & 51 & 52 & $2.12(0.89-9.51)$ & $2.69(1.01-9.66)$ & $0.05(-0.17-0.55)$ \\
\hline Positive & 54 & 2 & $2(3.85)$ & 50 (96.15) & 45 & 49 & $2(0.67-6.04)$ & $3.26(0.92-6.31)$ & $0.29(0-1.16)$ \\
\hline \multicolumn{10}{|l|}{ Localisation } \\
\hline PTB & 79 & 3 & $9(11.84)$ & $67(88.16)$ & 62 & 66 & $1.93(0.57-6.04)$ & $2.82(0.75-6.22)$ & $0.26(-0.12-0.80)$ \\
\hline EPTB & 40 & 0 & 5 (12.5) & 35 (87.5) & 34 & 35 & $2.29(1.23-10)$ & $2.95(1.15-10)$ & $0.06(-0.17-0.46)$ \\
\hline \multicolumn{10}{|l|}{ BCG } \\
\hline Negative & 6 & 0 & 2 (33.33) & $4(66.67)$ & 4 & 4 & $1.15(0.33-1.86)$ & $1.47(0.4-2.75)$ & $0.07(0.01-0.44)$ \\
\hline
\end{tabular}

Data are presented as $n, \mathrm{n}$ (\%) or median (interquartile range). IFN: interferon; TB: tuberculosis; PTB: pulmonary tuberculosis; EPTB: extrapulmonary tuberculosis; BCG: bacille Calmette-Guérin. \#: percentages are based on the total number of valid tests, excluding indeterminate results. 
a patient with meningeal tuberculosis receiving prednisone treatment. The sensitivity of the test slightly increases to $88.79 \%$ (95\% CI 81.41-93.47\%), when excluding immunocompromised patients from the data.

Six patients had a positive result by TB2 only. All these patients had smear-positive pulmonary tuberculosis (PTB) and four of them had more than three cavities. Moreover, we found that the difference between TB2 and TB1 was higher in smear-positive than smear-negative patients. Considering the difference between the two antigen tubes as a surrogate marker of the magnitude of $\mathrm{CD}^{+} \mathrm{T}$-cell responses, the last finding is in agreement with what observed in flow-cytometry studies. $M$. tuberculosis-specific $\mathrm{CD} 8^{+} \mathrm{T}$-cells correlate with an increased mycobacterial load and are more frequently found in smear-positive PTB individuals [11].

Finally, in the QFT-Plus test, we observed a significant difference in interferon- $\gamma$ response between the two tubes; however, whether this difference is due to the additional $\mathrm{CD} 8^{+} \mathrm{T}$-cell stimulation has not yet been proven. A previous study analysed the contribution of $\mathrm{CD} 4^{+}$and $\mathrm{CD} 8^{+}$antigen-specific responses to novel peptides with promiscuous human leukocyte antigen II binding capabilities derived from M. tuberculosis genes over-expressed in an in vitro macrophage model [12]. In the study by Losi et al. [12], a small subgroup of active tuberculosis patients had a significantly higher frequency of peptide-specific interferon- $\gamma$ $\mathrm{CD} 9^{+} \mathrm{CD}^{+}$and interferon- $\gamma \mathrm{CD} 69^{+} \mathrm{CD}^{+}$T-cells compared to controls. Flow-cytometry studies in a larger population need to be carried out to confirm the presence of $\mathrm{CD} 8^{+}$response in the newly added tube.

Despite progress being made in global tuberculosis control, the current incidence decline is far from what is needed to achieve tuberculosis elimination by the year 2050. Global tuberculosis control could be enhanced by the identification and treatment of symptom-free people who are latently infected with M. tuberculosis $[1,2]$. Of particular relevance for low-incidence countries would be the development of rapid and accurate tests able to detect, among subjects with LTBI, those at higher risk of developing active tuberculosis disease who would most benefit from preventive treatment [13].

QFT-Plus is a new IGRA that includes a set of peptides designed to stimulate M. tuberculosis-specific $\mathrm{CD}^{+} \mathrm{T}$-cells. The new test shows a high specificity and retains the same sensitivity as the previous version. In addition, the increased interferon $-\gamma$ release by combined stimulation of CD4 ${ }^{+}$and $\mathrm{CD} 8^{+} \mathrm{T}$-cells observed in the newly added antigen tube (TB2) might be advantageous for improving the assay's accuracy in patients with low $\mathrm{CD} 4^{+} \mathrm{T}$-cell counts.

○ @ERSpublications

QuantiFERON-TB Plus improves sensitivity for active TB and maintains high specificity among unvaccinated controls http://ow.ly/XjYPK

Lucia Barcellini ${ }^{1}$, Emanuele Borroni ${ }^{1}$, James Brown ${ }^{2}$, Enrico Brunetti ${ }^{3}$, Luigi Codecasa ${ }^{4}$, Federica Cugnata ${ }^{5}$, Paola Dal Monte ${ }^{6}$, Clelia Di Serio ${ }^{5}$, Delia Goletti ${ }^{7}$, Giulia Lombardi ${ }^{6}$, Marc Lipman ${ }^{2}$, Paola M.V. Rancoita ${ }^{5}$, Marina Tadolini ${ }^{8}$ and Daniela M. Cirillo ${ }^{1}$

${ }^{1}$ Emerging Bacterial Pathogens Unit, Division of Immunology and Infectious Diseases, IRCCS San Raffaele Scientific Institute, Milan, Italy. ${ }^{2}$ Royal Free London NHS Trust and UCL Respiratory, Division of Medicine, University College London, UK. ${ }^{3}$ Dept of Infectious Diseases, San Matteo Hospital Foundation-University of Pavia, Pavia, Italy. ${ }^{4}$ Regional TB Reference Centre, Villa Marelli Institute/Niguarda Ca' Granda Hospital, Milan, Italy. ${ }^{5}$ University Centre of Statistics for Biomedical Sciences (CUSSB), Vita-Salute San Raffaele University, Milan, Italy. ${ }^{6}$ Microbiology Unit - Dept of Experimental, Diagnostic and Specialty Medicine, S. Orsola-Malpighi University Hospital, Bologna, Italy. ${ }^{7}$ Translational Research Unit, Epidemiology Dept, National Institute for Infectious Diseases, Rome, Italy. ${ }^{8}$ Infectious Diseases Unit, Department of Medical and Surgical Sciences, Alma Mater Studiorum University of Bologna, S. Orsola-Malpighi Hospital, Bologna, Italy.

Correspondence: Daniela M. Cirillo, Emerging Bacterial Pathogens Unit, San Raffaele Scientific Institute, Via Olgettina 58, 20132 Milan, Italy. E-mail: cirillo.daniela@hsr.it

Received: Dec 032015 | Accepted after revision: Jan 012016 | First published online: Feb 122016

Support statement: We thank Qiagen (Germantown, MD, USA) for providing the QuantiFERON-TB Plus kits free of charge.

Conflict of interest: None declared.

Acknowledgements: The authors thank the study subjects for their generous participation, and G. Pellicciotta, P. Erba and I. Mascherona (IRCCS San Raffaele Scientific Institute, Health Care Staff Protection Unit, Milan, Italy) for their valuable support in control enrolment.

\section{References}

1 Diel R, Loddenkemper R, Zellweger J-P, et al. Old ideas to innovate TB control: preventive treatment to achieve elimination. Eur Respir J 2013; 42: 785-801.

2 Lönnroth K, Migliori GB, Abubakar I, et al. Towards tuberculosis elimination: an action framework for low-incidence countries. Eur Respir J 2015; 45: 928-952. 
Sester M, Sotgiu G, Lange C, et al. Interferon- $\gamma$ release assays for the diagnosis of active tuberculosis: a systematic review and meta-analysis. Eur Respir J 2011; 37: 100-111.

4 Pai M, Zwerling A, Menzies D. Systematic review: T-cell-based assays for the diagnosis of latent tuberculosis infection: an update. Ann Intern Med 2008; 149: 177-184.

5 Qiagen. QuantiFERON ${ }^{\star}$-TB Gold Plus (QFT-Plus) ELISA Package Insert. www.quantiferon.com/irm/content/PI/ QFT/PLUS/2PK-Elisa/UK.pdf

6 Rozot V, Vigano S, Mazza-Stalder J, et al. Mycobacterium tuberculosis-specific CD8+ T cells are functionally and phenotypically different between latent infection and active disease. Eur J Immunol 2013; 43: 1568-1577.

7 Nikolova M, Markova R, Drenska R, et al. Antigen-specific CD4- and CD8-positive signatures in different phases of Mycobacterium tuberculosis infection. Diagn Microbiol Infect Dis 2013; 75: 277-281.

8 Chiacchio T, Petruccioli E, Vanini V, et al. Polyfunctional T-cells and effector memory phenotype are associated with active TB in HIV-infected patients. J Infect 2014; 69: 533-545.

9 Lancioni C, Yendak M, Kiguli S, et al. $\mathrm{CD}^{+} \mathrm{T}$ cells provide an immunologic signature of tuberculosis in young children. Am J Respir Crit Care Med 2012; 185: 206-212.

10 Getahun H, Matteelli A, Abubakar I, et al. Management of latent Mycobacterium tuberculosis infection: WHO guidelines for low tuberculosis burden countries. Eur Respir J 2015; 46: 1563-1576.

11 Day CL, Abrahams DA, Lerumo L, et al. Functional capacity of Mycobacterium tuberculosis-specific T cell responses in humans is associated with mycobacterial load. J Immunol 2011; 187: 2222-2232.

12 Losi M, Knights AJ, Mariani F, et al. QuantiFERON-TB performance enhanced by novel Mycobacterium tuberculosis-specific antigens. Eur Respir J 2016; 47: 660-664.

13 D'Ambrosio L, Dara M, Tadolini M, et al. Tuberculosis elimination: theory and practice in Europe. Eur Respir J 2014; 43: 1410-1420.

Eur Respir J 2016; 47: 1587-1590 ｜ DOI: 10.1183/13993003.02033-2015 | Copyright @ERS 2016 